\title{
Maratona para a Popularização da Ciência da Computação na Educação Básica
}

\author{
${ }^{1}$ Michel Girotto Brum ${ }^{2}$ Marcia E. J. Kniphoff da Cruz, \\ 1,2 Departamento de Computação Curso de Licenciatura em Computação \\ Universidade de Santa Cruz do Sul - UNISC \\ Santa Cruz do sul - RS - Brasil \\ 1,2 michelgb@unisc.br e marciakniphoff@gmail.com ou mcruz@unisc.br
}

Resumo. Diferentes ações têm sido empreendidas para difundir o ensino de Ciência da Computação na Educação Básica. Com o objetivo de fomentar essa proposta, este relato apresenta a Maratona UNISC de Computação - MUC. A MUC é uma olimpíada científico/tecnológica que objetiva proporcionar novos desafios na área da Computação a estudantes de escolas de Ensino Fundamental e incentivar o interesse pela área. A primeira edição realizada no ano de 2015, oportunizou aos estudantes a resolução de desafios teóricos e problemas em linguagem de programação. Doze escolas da região do Vale do Rio Pardo e Taquari participaram. Uma nova edição está prevista para o ano de 2016 com a participação do Ensino Médio. Pretende-se ampliar a abrangência da MUC para outras regiões do país e incentivar a competição saudável na área da Computação, junto à Educação Básica.

Palavras chave: Scratch, Maratona, Computação.

Abstract. Different actions have been undertaken to spread the Computer Science education in basic education. In order to promote this proposal, this report presents the first Maratona UNISC de Computação - MUC. The MUC is a scientific/technological Olympiad which aims to provide new challenges in the field of Computing students from elementary schools and encourage interest in the area. The first edition held in 2015 , provided an opportunity to students to solve theoretical problems and challenges in programming language. Twelve schools in the Rio Pardo and Taquari Valley region participated. A new edition is scheduled for the year 2016 with the participation of high school. It is intended to expand the scope of MUC to other regions of the country and encourage healthy competition in computer area, next to the Basic Education.

Keywords: Scratch, Marathon, Computer.

\section{A CIÊNCIA DA COMPUTAÇÃO E A EDUCAÇÃO BÁSICA}

O desenvolvimento social e econômico de um país está diretamente relacionado ao seu potencial científico e tecnológico. No Brasil, mesmo com todos os esforços e ações para difundir o ensino da Ciência da Computação, observa-se que um há longo caminho a ser trilhado para efetivar estudos da área, na Educação Básica. Em análise dos artigos publicados nos anais do XXIII Workshop sobre Educação em Computação - WEI observa-se as grandes dificuldades do ensino da programação nos cursos de ensino superior, conforme citado por Barbosa (2011) e Correia (2015). Esses estudos, ainda, apontam dados alarmantes sobre a evasão dos cursos de Computação; o assunto tem se tornado pauta de encontros nacionais, segundo Massa (2015). Os problemas referentes podem ser equalizados com a introdução de estudos de Ciência da 
Computação na Educação Básica, através do desenvolvimento do Pensamento Computacional e de lógica para programação. Segundo Andrade et al (2013), o Pensamento Computacional se utiliza de atividades que introduzem os conceitos de coleta, análise e representação de dados, decomposição de problemas, abstração, automação, simulação, algoritmos e paralelismo, sem o uso de computadores. Em Ciência da Computação essas habilidades são básicas para o desenvolvimento posterior de algoritmos e programação.

Algumas escolas já estão incorporando o ensino de Computação nas suas rotinas diárias. O Scratch tem sido utilizado para o ensino da programação, por ser uma ferramenta visual e lúdica, mas poderosa. Permite a elaboração de algoritmos complexos. Outros trabalhos como os de Rabêlo (2015) e Batista (2015), relatam formas criativas e inovadoras de levar o ensino da computação de forma atrativa aos estudantes, utilizando Scratch, para elaboração de animações e jogos. A necessidade de oportunizar o desenvolvimento do Pensamento Computacional aos estudantes da Educação Básica está relacionada ao grau de complexidade dos problemas enfrentados no cotidiano. Ribeiro (2013), afirma que, noções matemáticas trabalhadas nas séries iniciais já foram conhecimentos apenas de intelectuais em séculos passados, contudo, hoje a programação de computadores está se tornando essencial.

Códigos de programação são partes integrantes de muitos dos recursos cotidianos, mas poucos tem o conhecimento par elaborá-los, o que justifica a necessidade emergente de repensar o currículo da Educação Básica do Brasil e atualizá-lo. É importante lembrar, segundo Ribeiro (2013), que o pensamento lógico-matemático difere do Pensamento Computacional; o raciocínio computacional utiliza a lógica como uma ferramenta no processo de resolução de problemas. Significa decompor os problemas em partes menores, para que possam ser resolvidos e combinados em função do resultado final, tornando o processo todo muito mais complexo que a simples tomada de decisão. Muitas escolas, ainda veem o ensino de informática apenas como a necessidade de dominar o uso de softwares e não como uma forma de desenvolver habilidades que estão se tornando necessárias, cada vez mais cedo, na sociedade, o que justifica a necessidade de incansáveis esforços para popularizar a Ciência da Computação na Educação Básica. Portanto, as iniciativas que promovem eventos com a finalidade de popularizar da Ciência da Computação são, cada vez mais, necessárias. Essas iniciativas incentivam as instituições de Educação Básica a incorporá-la em seus currículos regulares. Com a intenção de incentivar tais iniciativas, este artigo apresenta o desenvolvimento e os resultados de uma maratona de computação para estudantes das séries finais do Ensino Fundamental, que ocorre. A maratona ocorreu através de uma competição saudável ${ }^{1}$.

\section{OLIMPÍADAS CIENTÍFICAS}

Olimpíadas cientificas objetivam difundir o ensino da ciência e da tecnologia e identificar talentos em potencial, para incentiva-los a seguir carreiras nas áreas de ciência e tecnologia. No Brasil, as olimpíadas tiveram seu início em 1978 e desde 2002 vem recebendo apoio do poder público. Hoje, diversas olimpíadas cientificas como a Olimpíada Brasileira de Robótica - OBR, Olimpíada Brasileira de Informática - OBI são fomentadas por órgãos como o Conselho Nacional de Desenvolvimento Científico e Tecnológico (CNPq) e Ministério da Educação em parceria com a Fundação Nacional de Desenvolvimento da Educação (FNDE/MEC). Alguns

\footnotetext{
${ }^{1}$ A competição saudável incentiva o desafio como forma de superação pessoal e a disputa com demais colegas torna-se um benefício comum, pois os resultados da competição incentivam a busca por mais conhecimento.
} 
eventos como a OBI utilizam os resultados das provas na modalidade teórica para selecionar e formar equipes que irão representar o Brasil em outras olimpíadas de nível internacional, como a International Olympiad in Informatics - IOI. A primeira IOI foi proposta na vigésima quarta conferência da United Nations Educational, Scientific and Cultural Organization (UNESCO), em Paris no ano de 1987, que também patrocinou a primeira IOI, realizada na Bulgária em 1989. Desde então, outras edições vêm acontecendo anualmente, sendo que, a cada ano a IOI é sediada em um país diferente. As tarefas nessas olimpíadas versam sobre algoritmos e desafios em tecnologias de informação. No caso da IOI, versam também sobre análise de problemas, estruturas de dados e design de algoritmos. Além dos eventos nacionais, diversas instituições de nível superior promovem olimpíadas científicas regionais voltadas à Educação Básica.

\section{MARATONA UNISC DE COMPUTAÇÃO}

A Maratona UNISC de Computação - MUC foi promovida pelo curso de Licenciatura em Computação e Projeto UNISC Inclusão Digital ${ }^{2}$. Aconteceu na Universidade de Santa Cruz do Sul - UNISC e envolveu escolas de Educação Básica do Vale do Rio Pardo e Taquari. A MUC teve por objetivos:

- Proporcionar novos desafios na área da Computação a estudantes de escolas de Educação Básica e de organizações educativas.

- Incentivar o interesse pela área da Computação, introduzindo o pensamento lógico, através da resolução de problemas e de técnicas de programação de computadores.

- Identificar e premiar talentos na área da Computação e incentivá-los a seguir carreiras nas áreas de ciência e tecnologia.

No primeiro ano, a MUC aconteceu em duas modalidades: Nível 1 e Nível 2, contando ambas as modalidades com dois desafios. O primeiro desafio foi a resolução da atividade "circuito de questões" realizado no turno da manhã. O segundo, a resolução de atividade em laboratório de informática, no desenvolvimento de "Desafios de Programação", com tarefas apropriadas para cada modalidade, no turno da tarde. Para a próxima edição, estuda-se a ampliação para três níveis, com o objetivo de envolver também o público do Ensino Médio. No Nível 1, as questões do circuito versaram sobre problemas de Lógica e Computação. $\mathrm{Na}$ atividade desafio de programação, as duplas receberam programas já criados, devendo montar os algoritmos para que o programa funcionasse, conforme objetivo indicado nas tarefas, utilizando a linguagem visual Scratch 2. No Nível 2, as questões do circuito versaram sobre problemas de Lógica e Computação. Na atividade desafio de programação, as duplas recebem enunciados de programas, tendo como desafio desenvolver algoritmo e a interface para que o programa funcionasse.

Foi desenvolvido um hotsite para a divulgação da MUC, inscrições e divulgação dos resultados. Para a elaboração dos formulários de inscrição, foi utilizado a plataforma web JotForm, que permite a integração de formulários web em qualquer site. $\mathrm{O}$ site da MUC

\footnotetext{
2 Foram apoiadores da MUC, ainda: Núcleo de Socialização Ciência e Tecnologia - NTSC, Programa UNISC Escola, Projeto de Pesquisa $\mathrm{O}$ uso de estilos cognitivos e agentes pedagógicos no processo de ensino-aprendizagem em sistemas tutores inteligentes e Subprojeto de Informática PIBID/UNISC.
} 
encontra-se disponível na Internet e foi desenvolvido utilizando a plataforma web gratuita Weebly. A maratona aceitou equipes formadas por duplas de alunos, acompanhadas por professor responsável. Para popularizar o evento não foi estimulado limite de inscritos por escola nem por professor orientador, que pode inscrever alunos de mais de uma modalidade ou escola.

\subsection{Elaboração das atividades}

Para a elaboração das atividades, realizou-se um estudo das últimas cinco edições da OBI e OBR, baseando-se nos assuntos abordados nessas provas de modalidade teórica. As questões não envolvem conteúdos que não estejam em livros ou que não sejam abordados na escola, mas explora a capacidade dos competidores em decompor um problema maior e resolvê-lo, abstrair e apresentar uma solução.

A MUC foi dividida em três partes distintas: O circuito de questões, a tarefa cultural e os Desafios de Programação. A primeira, aconteceu na parte da manhã, em auditório, com espaço adequado. A segunda em laboratórios de informática no período da tarde. Ambas as partes tiveram duração de três horas. Além das provas, o evento alocou um tempo de duas horas para o intervalo entre o Circuito de Questões e os Desafios de Programação. O amplo tempo livre foi dedicado para a integração entre os participantes. Nesse momento, realizaram uma tarefa extra denominada "Tarefa Cultural", com um propósito lúdico, que foi liberada ao final da manhã.

Para a elaboração do edital e das questões e desafios, reuniu-se um comitê organizador, sendo este composto por estudantes do curso de Licenciatura em Computação, integrantes do diretório acadêmico e professores do Departamento de Computação.

\subsubsection{Circuito de questões}

O circuito de questões foi composto por três provas. Cada uma das provas iniciou com uma situação problema com cinco alternativas de respostas, sendo apenas uma correta. O primeiro desafio para o estudante foi interpretar e entender o problema. Para a resolução precisaram escolher uma abstração, empregar lógica e algoritmos. Cada prova foi entregue em horário prédeterminado, sendo que, para o recebimento da próxima prova, a dupla deveria entregar no ato a prova anterior, com sua resolução. Para cada nível, foram desenvolvidas provas distintas, com diferentes graus de dificuldade. A Figura 1 apresenta uma questão teórica do circuito de questões: 
DESAFIO 2 (20 PONTOS)

\section{Baralho Digital}

Ana e Bernardo criaram um novo jogo usando um baralho. No novo jogo, o baralho deve conter 48 cartas, igualmente divididas entre pretas e vermelhas. Nesse jogo, as cartas são dispostas na mesa viradas para baixo, e cada jogador deve virar uma carta por vez. Ao virar a carta, ele retira da mesa e conta a pontuação de acordo com o algoritmo ao lado. Uma rodada completa acontece quando os dois jogadores retiraram uma carta. Para definir o número de rodadas um dos participantes deve jogar 4 dados comuns na mesa e somar os valores que ficaram para cima dos 4 dados.

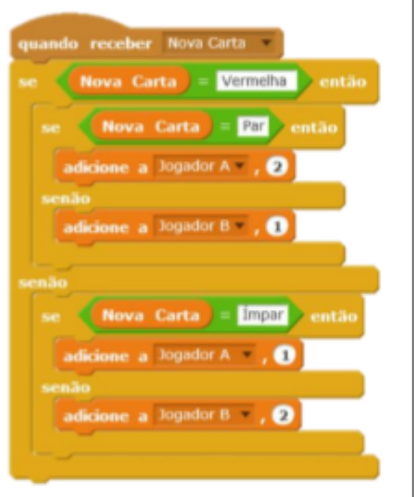

Figura 1. Exemplo de questão teórica presente na prova do circuito de questões

Na Figura 1 pode-se observar uma situação problema, na qual é necessário aplicar um algoritmo para responder corretamente a perguntas. Para a correta resolução, os estudantes precisaram empregar habilidades relacionadas ao Pensamento Computacional. A pontuação foi dada em função de condições estabelecidas previamente, nas regras.

\subsubsection{Tarefa Cultural}

A Tarefa Cultural solicitou que os participantes fotografassem frases pintadas em prédios da universidade e publicassem no modo público, em redes sociais online, marcadas com hashtags \#MUCÉShow. As hashtags são utilizadas em meios de publicação com a finalidade de organizar e agrupar conteúdo durante a busca pelos usuários, servindo como filtro. Essa tarefa teve importante papel na divulgação do evento. A Figura 2 apresenta um exemplo de fotografia:

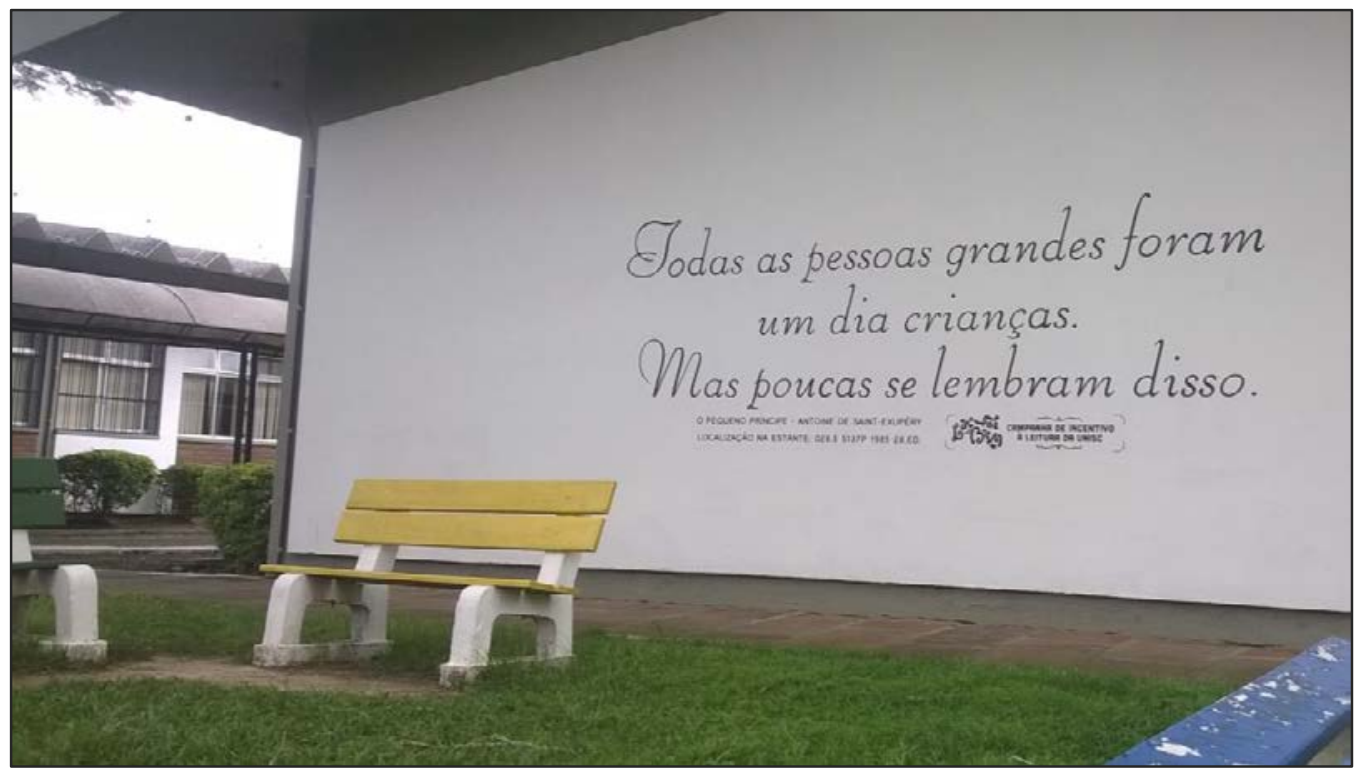

A Figura 2 apresenta exemplo de fotografia esperada dos estudantes 


\subsubsection{Desafios de Programação}

Para a resolução dos Desafios de Programação, cada dupla teve à disposição um computador, em laboratório de informática, com o software Scratch 2.0, ferramenta de programação em linguagem visual desenvolvida pelo Massachusetts Institute of Technology - MIT. A Figura 2 apresenta uma questão do Desafios de Programação:

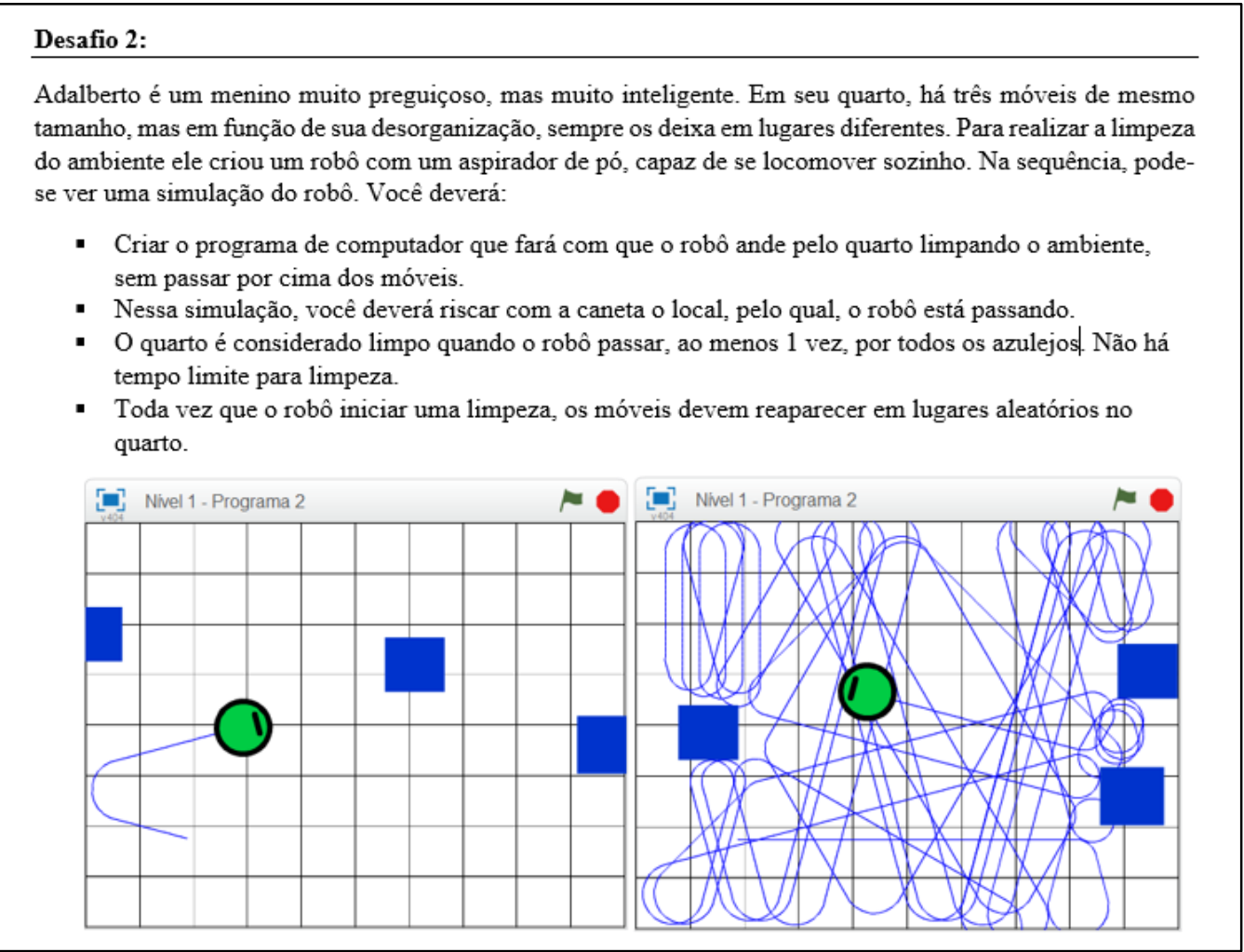

Figura 2. Desafio da sessão “Desafios de Programação”, apresentado nos dois níveis da MUC

Esse desafio e exigiu dos participantes conhecimento sobre loop, condicionais, operadores lógicos, aritméticos e sensores. Para cada nível o desafio 1 apresentou grau de dificuldade menor, envolvendo programação, apenas de movimento e comandos de aparência para criar animações. No último desafio o grau de complexidade e dificuldade foi elevado. Para a resolução foram necessárias habilidades para trabalhar com dados de entrada do usuário. No caso do Nível 1 o desafio envolveu operações matemáticas com variáveis e tomadas de decisões. No caso do Nível 2 operações lógicas e aritméticas envolvendo listas de dados, tomadas de decisões e noções de estrutura de dados.

\section{RESULTADOS, AVALIAÇÃO E PROPOSIÇÕES FUTURAS}

Participaram da MUC doze escolas públicas e privadas, e instituições educacionais, quinze professores da Educação Básica, dezesseis bolsistas e alunos do curso de Licenciatura em Computação e cinco professores do Departamento de Computação, além dos cento e seis estudantes envolvidos nas oito horas de maratona.

Todas as provas foram corrigidas por três integrantes do comitê avaliador. O Quadro 1 apresenta o cronograma da MUC e avaliação: 


\begin{tabular}{|l|l|l|l|}
\hline Atividade & Início & Fim & Pontuação \\
\hline Abertura e Validação da Inscrição & 8 horas & 9 horas & - \\
\hline Problema 1 & 9 horas & 10 horas & 20 \\
\hline Problema 2 & 10 horas & $10 \mathrm{~h} 45 \mathrm{~min}$ & 15 \\
\hline Problema 3 & $10 \mathrm{~h} 45 \mathrm{~min}$ & $11 \mathrm{~h} 30 \mathrm{~min}$ & 15 \\
\hline Tarefa cultural & $11 \mathrm{~h} 30 \mathrm{~min}$ & $13 \mathrm{~h} 30 \mathrm{~min}$ & 5 \\
\hline Intervalo para Almoço & $11 \mathrm{~h} 45 \mathrm{~min}$ & $13 \mathrm{~h} 30 \mathrm{~min}$ & - \\
\hline Desafio de Programação em Scratch & $13 \mathrm{~h} 30 \mathrm{~min}$ & $16 \mathrm{~h} 30 \mathrm{~min}$ & 45 \\
\hline Envio da Atividade de Programação & $16 \mathrm{~h} 30 \mathrm{~min}$ & 17 horas & - \\
\hline
\end{tabular}

Quadro 1: Horários e pontuação das tarefas da MUC

Ao final da maratona, a soma das tarefas foi de 100 pontos, distribuídos em 50 pontos para Circuito de Questões, 5 pontos para Tarefa Cultural e 45 pontos para os Desafios de Programação. Após a avaliação das 61 duplas participantes, constatou-se a grande heterogeneidade dos estudantes, relativa aos conhecimentos de lógica de programação e Pensamento Computacional. A Figura 3 apresenta dados: 49\% dos participantes não obtiveram 25 pontos; apenas $20 \%$ dos participantes tiveram mais de 50 pontos, conforme apresenta a Figura 3:

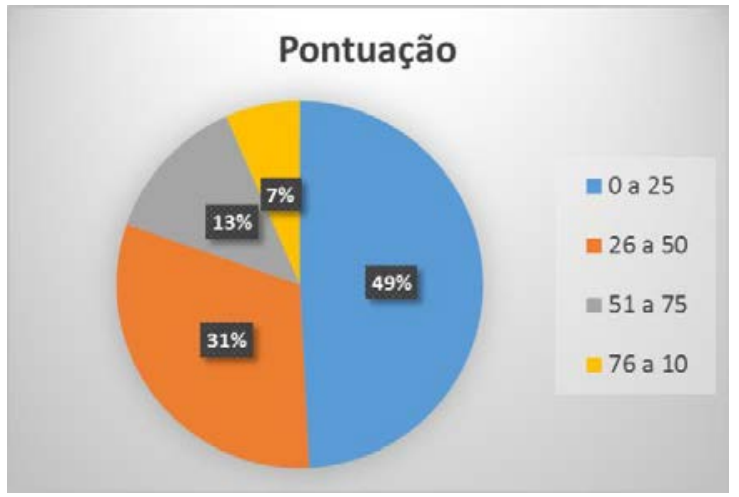

Figura 3: Porcentagem de estudantes por faixa de pontuação

O resultado da primeira edição demonstra a necessidade de uma atualização no currículo escolar; há urgência em inserir Pensamento Computacional e técnicas de programação junto ao ensino de informática nas escolas de Educação Básica.

$\mathrm{Na}$ avaliação dos professores orientadores das escolas, a maratona foi muito positiva. Consideraram a duração do evento apropriada. Quanto ao tempo disponível para a resolução das atividades, $67 \%$ indicaram ter sido apropriado para as tarefas, $33 \%$ indicaram que para o tempo disponível as tarefas poderiam ter sido maiores. Quanto ao grau de dificuldade das tarefas, 67\% indicaram ter sido apropriado para o nível dos estudantes, e 33\% indicaram que a dificuldade foi alta. Foi questionado também o grau de satisfação geral dos participantes quanto à organização do evento. Neste quesito, solicitou-se a atribuição de uma nota de 1 a 5 , sendo 1 para muito insatisfeito, e 5 para muito satisfeito. Conforme a Figura 5, a maior parte atribuiu nota 4. 


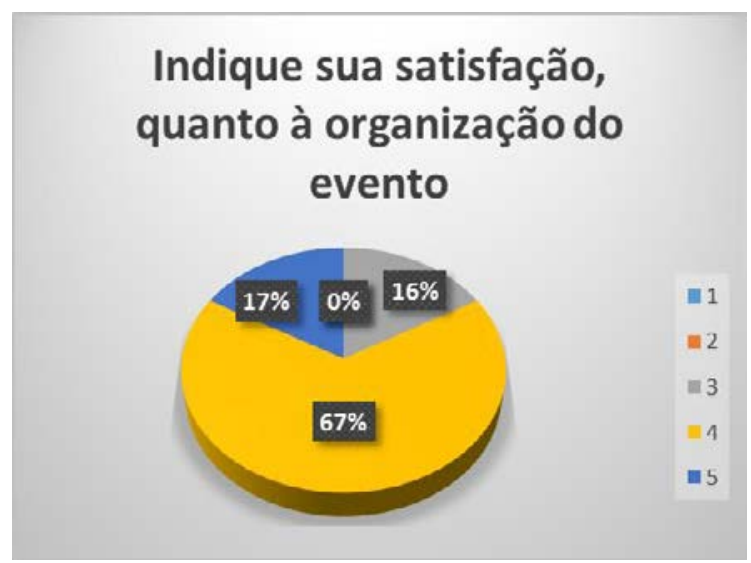

Figura 5: Pesquisa com professores e responsáveis

Durante o intervalo os organizadores conversaram com os alunos participantes, professores e responsáveis, a fim de ouvi-los sobre o andamento da maratona. Os participantes descreveram o evento de forma muito positiva. Entre os aspectos mais importantes foi citada a integração com alunos de outras escolas que também aprendem computação, os desafios de trabalho em conjunto, a proximidade dos desafios com o mundo real. Também relataram terem visto o evento como uma forma de incentivo ao aprendizado de programação, pouco enfatizado na maioria das escolas. "Foi uma experiência muito divertida que me ensinou a trabalhar em equipe e me motivou a continuar aprendendo sobre programação", comentou um estudante. Para um dos ganhadores, "[...] foi uma maratona que me ensinou duas coisas importantes, que não importa se ganhamos ou perdemos, e a trabalhar em equipe com minha dupla". Durante a premiação uma campeã acrescentou que "a MUC foi um grande evento educacional, onde muitos alunos tiveram a oportunidade de participar e acrescentar conhecimento sobre algo tão interessante como a computação".

Com a realização da MUC, conclui-se que a oferta de competição saudável é uma excelente estratégia educativa, no que se refere ao incentivo do Pensamento Computacional para a Educação Básica. Os estudantes mostraram muito interesse em ampliar conhecimentos de programação; os professores sensibilizaram-se sobre a importância do ensino de computação para a formação dos alunos.

Como proposição futura pretende-se estender a participação para o Ensino Médio e gradativamente, ampliar o grau de dificuldade dos desafios e exigências na programação. Pretende-se, ainda, ampliar a divulgação científica nos meios de comunicação de massa para incentivar o ensino da computação na Educação Básica.

\section{Referências}

Batista, W. P. et. al. (2015). Oficinas de Aprendizagem de Programação em uma Escola Pública através do Ambiente Scratch In: Anais do XXIII WEI. SBC.

Barbosa, L. S., Fernandes, T. C., and Campos, A. M. (2011). Takkou: Uma ferramenta proposta ao ensino de algoritmos. In Anais do CSBC/XIX WEI. 
Correia, A. L. et. al. (2015). Uso de avaliação por pares em disciplinas introdutórias de programação. In Anais do CSBC/XXIII WEI.

International Olympiad in Informatics - IOI, acessado em 04 de março de 2016, disponível em http://www.ioinformatics.org/history.shtml

JotForm. Disponível em http://www.jotform.com/. Acesso em abril de 2016.

Massa, S. Monica. (2015). A formação didático-pedagógica do docente da área de computação: um estudo de caso em uma Universidade Brasileira. In: Anais do XXIII WEI. SBC.

MUC. Edital Maratona UNISC de Computação - MUC, 2015. Disponível em: http://imuc.weebly.com/. Acesso em abril de 2016.

Rabêlo, H. M. et al. (2015). Scratch na produção de recursos interdisciplinares com disciplinas indígenas. In: Anais do XXIII WEI. SBC.

Ribeiro, Leila. (2013) Painel sobre "O Pensamento Computacional no Ensino Fundamental e Médio" realizado em outubro de 2013 no II Workshop-Escola de Informática Teórica (WEIT). Instituto de Informática / UFRGS

SCRATCH 2. Disponível em scratch.mit.edu. Acesso em dezembro de 2015. 